SRC TR 88-6

Functional Design of an Integrated CIM System at the Facility Level

by

G. Harhalakis, M.E. Ssemakula, and A. Johri 


\title{
Functional Design of an Integrated CIM System at the Facility Level
}

\author{
G. Harhalakis ${ }^{1}$, M.E. Ssemakula, A. Johri ${ }^{1}$ \\ Systems Research Center \\ and Department of Mechanical Engineering \\ University of Maryland, College Park, MD 20742.
}

1 supported in part by the NSF under grant CDR-85-00108 


\title{
FUNCTIONAL DESIGN OF AN INTEGRATED CIM SYSTEM
}

AT THE FACILITY LEVEL

\author{
By \\ G. Harhalakis, M.E. Ssemakula, A. Johri \\ Department of Mechanical Engineering, \\ University of Maryland \\ College Park, MD 20742, U.S.A.
}

\begin{abstract}
Computer Integrated Manufacturing (CIM) is often understood as simply the integration of $C A D$ and $C A M$. In fact it is much more than this. It is the systems approach of linking together the various automation tools available today, so as to enable the control of an entire manufacturing operation, as well as related business functions. Unlike most of the recent research in CIM, we are not addressing the integration of hardware and software on the shop floor. Instead, we consider that Manufacturing Resource Planning (MRP-II), has the best inherent features for the linking of the various high level manufacturing functions. In this work, integration centers around parts specifications, routings and the workcenters in the system. A model for this integration at the facility level, is presented along with the rules of interaction between the systems. The model is based on the principle of database interoperability, and will use a formal language named 'Update Dependencies' for the implementation.
\end{abstract}


1. INTRODUCTION.

Feeling the pressure from foreign and domestic competitors, many US and European companies have had to adopt some of the many advanced technologies available today, which automate individual functions of a manufacturing facility. Some of these technologies are: Computer Aided Design (CAD), Computer Aided Manufacturing (CAM), Manufacturing Resource Planning (MRP-II), Flexible Manufacturing systems (FMS), Group technology (GT), and Computer Aided Process Planning (CAPP), to name just a few. There is no doubt that these technologies, when implemented correctly, do help in promoting efficiency, within the specified area. When they are collectively viewed as a system, however, overall efficiency leaves much to be desired. The various automated areas are very closely related to one another. For example CAPP takes geometric data from CAD as its input and generates a detailed process plan which in turn is used by CAM and MRP-II to control shop floor operations. Given these intimate relationships between the areas, it was visualised that, if some of these could be interfaced with one another, the resulting efficiency and productivity would be greatly enhanced. This interfacing, or the rule-based automated communication, within related areas of a modern manufacturing facility, is the basis of Computer Integrated Manufacturing.

Before the advent of the CIM concept, there was a proliferation of heterogenous hardware, and software, which optimised each function with a very narrow area of focus. As is also common these days, due to lack of any set standards, scores 
of different pieces of software became available for each functional area, each formatting its database differently. So the situation as it stands today is that different companies have different software for automating the same areas. This factor by itself makes up the major part of the barrier towards CIM. The amount of capital invested in existing software and hardware neccesitates modular and piecemeal implementation of an integrated system. Since the same areas have different existing software, with different data formats, one CIM implementation effort which works irrespective of the kind of software used, is not feasible. Instead, rules for integration will have to be developed for generic systems, along with the complete implementation models for such systems. This will serve as the general means for CIM. This generic CIM model, with minor modifications, depending upon existing software in a manufacturing plant, will result in an actual working CIM facilty.

\section{THE CIM MODEL}

It is proposed that MRP-II should serve as the coordinating and controlling medium of all of these technologies. This is illustrated in Figure 1.

This is because MRP-II alone has the position of posessing data common to most of the computer aided tools available today. So any kind of integration effort should begin with MRP-II as the hub of an integrated system (Fox 1984).

Although the concept of CIM has been loosely defined, neither a thorough system architecture, nor a requisite 
integration vehicle is yet available. There are many problems and issues which must be resolved, before CIM is possible, and the question of database architecture is of the utmost importance (Melkanoff 1984). There are two approaches to designing a database architecture. The first is a 'general database', which is a single database, maintaining all the system data, and is accessible by all of the system functions. The second is a system of multiple databases, each function having its own database, with interoperability capabilities, for effective communication with other databases. The latter approach is advocated here for a variety of reasons, the primary one being that considerable capital has already been invested in existing software and hardware. It would not be economically feasible to design the entire CIM system "from scratch". Where possible, the integration efforts should aim at utilizing existing systems.

A prototype model for the integration of MRP-II and CAD has already been established and implemented using the principles of multidatabase interoperability (Harhalakis 1986, 1987). This work deals with the continuation of this integration effort by adding Computer Aided Process Planning as a seperate function in the system. Computer Aided Process Planning is an essential component of a truly integrated system (Ssemakula 1987). The role of CAD is to facilitate the design of parts and assemblies, where assemblies are really just arrangements of component parts. Ideally, CAPP is visualised as influencing the design process, by evaluating the manufacturability of the designed part. It also is solely responsible for the generation of the process plan, 
which is a set of detailed instructions for manufacturing the part. Within the system, MRP-II plays the role of cataloging each part and assembly under the part master record, and recording the product structures. It also stores the process plan in its routings module, handles the addition and deletion of workcenters in the system, and issues shop orders based on the master production schedule and associated routings. So CAD and CAPP are viewed as the two design centers and MRP-II is restricted to the role of an execution function alone.

In our earlier work, the process planning activity was included in MRP-II, within the product and process definition module (Harhalakis 1986). This was an unsophisticated form of process planning, and not the way a real manufacturing system handles such an activity. In reality, the process planning department finalises the process plan, and this plan is recorded in the routings module and passed down to the shop floor in the form of a shop packet. The MRP-II/ CAD/ CAPP integrated system restricts MRP-II to such a role, and attempts to automate the information flow between CAPP and the MRP-II routings module. This eliminates the errors and delays associated with manual entry of process planning data into MRP-II. In addition, since MRP-II is the center for addition and deletion of workcenters, and CAPP keeps a record of workcenter details in its workcenter database, the flow of information regarding workcenters is integrated into the system.

The data commonality between CAD and MRP-II centers around product definition, whereas between CAPP and MRP-II, it centers around routings and workcenter records. Figure 2 shows the nature 
of information flow between the different functions. We have part and revision information flow between MRP-II and CAD; part, revision and routing information flow between CAD and CAPP; and routings and workcenter information flow between CAPP and MRP-II.

\section{FUNCTIONAL DESIGN}

The integrated system is centered around the data commonality between the three different functions. Specifically, the data commonality is related to the part records, the part revision records, the routing records, and the workcenter records. The following represent the data records maintained by each function.

\section{$\underline{\text { PART }}$ RECORDS}

CAD

Part Number

Drawing Number

Drawing size

Description

B.O.M. Unit of Measure

$-$

$-$

$-$

$-$

Supersedes Part Number

superseded by Part Number

$$
\text { MRP-II }
$$

Part Number

Drawing Number

Drawing Size

Description

B.O.M. Unit of Measure

Purch/Inv Unit of Measure

U.O.M. Conversion Factor

Source Code

Standard cost

Lead Time

Supersedes Part Number

Superseded by Part Number

\section{PART REVISION RECORDS}

CAD

Part Number

Revision Level

Effectivity start Date

Effectivity End Date

Status code

Drawing File Name
MRP-II

Part Number

Revision Level

Effectivity start Date

Effectivity End Date

Status code 


\section{ROUTING RECORDS}

CAPP

Routing Number

Part Description

Unit of Measure

operation Number

operation Description

Work Center I.D. Number

set up time

Machining time

Handling time

Run Time

Feed

Speed

Depth of cut

Number of Passes

-

$-$

-

Status code
MRP-II

Routing Number

Part Description

Unit of Measure

Operation Number

operation Description

Work Center I.D. Number

set up time

-

Run Time

$-$

-

$-$

Resource code

Begin Date

End Date

status Code

\section{WORKCENTER RECORDS}

$$
\text { MRP-II }
$$

I.D. Number

Description

Department

Capacity (hours)

Rate code

Resource Capacity

Dispatch Horizon

Effectivity start Date

Effectivity End Date

Status code

-

-

$-$

-

$-$

$-$

-

-

$-$

-

-
CAPP

I.D. Number

Description

Department

-

Effectivity start Date Effectivity End DAte

Status Code

Horse Power

Speed Range

Feed Range

Work Envelope

Accuracy

Tool Change Time

Feed Change Time

Speed Change Time

Table Rotation Time

Tool Adjustment Time

Rapid Traverse Rate 
It must be noted that within a system two different parts cannot be represented with the same part number. Conversely, different part numbers should not be assigned to the same part. However, a part can have a number of revisions coexisting in the system, only one of which is active at any time.

\subsection{STATUS CODES}

The working of the model can be represented by examining the status codes associated with each entity in the system (ie. part routing and workcenter).

\section{1 .1 PART REVISION STATUS}

\section{CAD}

W - 'working'; At a conceptual or preliminary stage, prior to approval, and not transmittable to MRP-II.

$\mathrm{R}$ - 'Released'; An active Part, whose design has been approved and finalised.

H - 'Hold'; Under review, pending approval, possibly with a new revision level. The part should not be used by either system.

o - 'obsolete'; The part is obsolete.

\footnotetext{
MRP-II

R-'Released'; An Active Part, whose purchase or manufacture can be initiated in MRP-II.

$\mathrm{H}$ - 'Hold'; Not to be used by MRP-II.
} 


\section{CAPP}

W - 'Working'; At a conceptual or preliminary stage, prior to approval.

R - 'Released'; An active part, whose design has been approved and finalised

H - 'Hold'; Under review, pending approval, possibly with a new revision level.

o - 'Obsolete'; The part is obsolete.

\section{CAPP}

W - 'Working'; At a conceptual or preliminary stage, prior to approval, and not transmittable to MRP-II.

R - 'Released'; An active routing. Finalised and approved.

$\mathrm{H}$ - 'Hold' ; Under review, pending approval, possibly with a new revision level. The routing should not be used by either system.

o - 'obsolete'; The routing is obsolete.

\section{$\underline{\text { MRP-II }}$}

R - 'Released'; an active routing, able to be passed down to the shopfloor, by MRP-II.

H - 'Hold'; Not to be used by MRP-II. 


\section{1 .3 WORKCENTER STATUS}

$\underline{\text { MRP-II }}$

R - 'Released'; An active workcenter.

H - 'Hold'; Not to be used by MRP-II.

D - 'Deleted'; A workcenter deleted from the system.

\section{CAPP}

W - 'Working'; At a preliminary stage, workcenter details yet to be entered in CAPP workcenter record.

$\mathrm{R}$ - 'Released'; An active workcenter, able to be used in process plans.

H - 'Hold'; Under review, not to be used for process plans.

o - 'Obsolete'; The Workcenter is obsolete.

\subsection{MODEL DESCRIPTION}

The integrated system is made up of a number of likely scenarios. For example, a new part being introduced into the system from any one of the constituent functions, will have to be treated differently from the case of another new part being introduced from some other function. This is because each of the functions has a role to play in assimilating the part in the system, and so the structure and the sequence of required events change, depending upon where the part originates. It has even been found necessary to develop different scenarios, depending on whether the part is a manufactured part, or a purchased one. Given below is a summary of the scenarios which describe the model. 


\section{PARTS}

(i) New manufactured product part from CAD

(ii) New purchased product part from CAD

(iii) New manufactured tool part from CAPP

(iv) New purchased tool part from CAPP

(v) New purchased supply part from MRP-II

ROUTINGS

(i) Generated in CAPP; included in above scenarios (i, and iii) for the purpose of discussion.

(ii) Revision of routings

WORKCENTERS

(i) Installation of a new workcenter

(ii) Deletion of workcenter

(iii) Reviewing a workcenter in CAPP

(iv) Reviewing a workcenter in MRP-II

REVISIONS

(i) Revisions of manufactured product parts from CAD

(ii) Revisions of purchased product parts from CAD

(iii) Revisions of manufactured parts from CAPP

(iv) Revisions of purchased parts from CAPP

(v) Revisions of purchased supply parts from MRP-II

(vi) Revisions of purchased as well as manufactured product parts from MRP-II.

It is not possible to discuss each of these scenarios, due to space limitations. However, a sample of different types of scenarios will be described. 
being worked on, and CAPP will be called upon to contribute to the design, in terms of clearing the design from the point of view of manufacturability, and in finalising the product structure in case of assembled parts. After CAD and CAPP work on the part, and the design is ready in every aspect, it is released in CAD. (N.B. The actual detailed interaction between CAD and CAPP regarding the design is outside the scope of this integrated model, however it is visualised that the geometric part data is downloaded to CAPP from CAD, and then some form of interaction is required in finalising the design). CAPP may not be ready to release the routing at this point, as its process plans may not be finalised yet. CAPP should check that the part has been released in $C A D$, before giving the routing a released status. The reasons for this are explained later.

As soon as CAD releases the new part, several actions are initiated. A skeletal part master record for the new part is established in MRP-II. Those data fields maintained in MRP-II, but not in CAD, are initiated as unknown, until supplied by MRPII users.

Second, a revision record is established in MRP-II, for the new part (state 1d), using the part number, and revision level from the CAD revision record. The effectivity dates are once again left unknown. The status of MRP-II revision is set to hold, since many of the fields required by the MRP-II system, have been initialised to unknown, and have to be completed before MRP-II can consider the part to be active. MRP-II also has to wait for CAPP to release the routings, as its lead time information for manufactured parts comes from its production routings, and these 
are automatically updated to it by CAPP when it releases the routings. Since CAPP releases the routings after CAD releases the part, MRP-II has to have the status as hold. The reason that CAPP releases the routings after CAD releases the part, is that finalised process plans are normally completed only after the design is finalised. For example the jigs, fixtures and tools required for the part have to be finalised in CAPP after the design is complete. Also, MRP-II must have the part established in its part master record, before it can accept the routings from CAPP.

The third action to be initiated, is that CAD checks for a value in the 'supersedes part number' field of the CAD part record. If it finds a valid part number in this field, the part number of the new part is inserted into the 'superseded by part number' field of the CAD part record, of the part number found. The MRP-II part master record is likewise modified to reflect the supersession. The latest revision of the part being superseded, which may have a released status (state $2 \mathrm{a}$ ), or which may be on hold (state $2 \mathrm{~b}$ ), is then made obsolete (state $2 \mathrm{c}$ ). If all the preceding steps are successful, the status of the new CAD revision is changed to released (state 1c).

MRP-II now starts working on the part record of the part in its database. It fills in whatever required information is available, and also waits fot CAPP to release the routings, before it can proceed any further.

When CAPP has finalised its process plan, the routing is given a released status. Once again a series of consistency 
checks are initiated before the release can be successful.

At this point, a check is made to make sure the part for which the routing is to be released has a released status in CAD. If it does not, a message to this effect is generated in CAPP, and the release is not successful. Then a check is made, to make sure that the effectivity dates of all the workcenters in the routings are at least six months (or some other applicable time period) ahead. If this is not done, the routing may become inapplicable before the product can be produced. Checks are also made to ensure that the essential data fields in the CAPP routing are complete. If all these checks are successfully made, the routing release is successful and then the routing gets a released status, (state 1e), and is updated immediately to the routings module of MRP-II, with a status of hold (state If).

As explained earlier when $C A D$ enters a part into the system, (in working status) by establishing the part number, unit of measure, and description, the same update takes place in CAPP also in working status, which becomes the foundation for a routing. Since different revisions of a part will have different routings, the routing identification number will start with Part number/Revision number. However since more than one routing can exist for any part, a particular routing is identified by the Part number/Revision number, and a qualifier which identifies that particular routing. This qualifier contains both the routing alternative number, and the routing revision number. This enables the user to incorporate additional revisions for each routing. The additional numbers are added to the original part number by CAPP. The MRP-II routings module identifies a routing by part 
number/ Revision number/Alternative number/Routing revision number also.

The CAPP routing record with its major data elements creates a skeletal routing record in MRP-II. As stated earlier, the routing status is first set to hold in MRP-II, since the MRP-II users have to complete certain data fields, such as resource codes, and effectivity dates, before the routings can be considered active.

The final step in this chain of events, is that after MRP-II has finalised the relevant records, it gives the part a released status (state $1 \mathrm{~g}$ ).

Similarly, scenarios for new parts from CAPP as well as MRPII can be developed, with modifications in the sequence of events. New purchased parts originating from either CAD, or CAPP would not involve the creation of routings. Finally, MRP-II can originate only purchased parts of the nature of office supplies and maintenance materials.

\section{2 .2 INSTALLATION OF A NEW WORKCENTER}

Consider the case where a workcenter is installed in the system, whose status diagram is shown in figure 4. The installation of a new workcenter always originates in MRP-II. Initially, the new workcenter to be added is on hold in MRP-II. This is the stage where the finalisation of all the details takes place. eg;

- I.D. Number

- Description

- Department

- Capacity (hours) 
- Rate code

- Effectivity Dates

- Resource Capacity

- Dispatch Horizon

This is represented by state ' $a$ ' in the status diagram.

When MRP-II has all the information it needs, to record the workcenter, it gives the workcenter a released status. At this point, checks are made to see if all the necessary fields are complete. If any of them have been left blank, the system gives a message to that effect, and the release is not successful. Another check is done to see if the workcenter ID number already exists. If it does, once again the release is not successful. If the above checks are successful, then the release is successful (state b) and it in turn triggers the establishment of the following attributes, in the workcenter record in CAPP (state c).

- I.D. Number

- Description

- Department

- Effectivity dates

This update to CAPP is automatically given a status of working. This notifies CAPP users that a new machine exists in the facility, and information necessary for its incorporation in the CAPP workcenter file, should be input. The additional data are as follows.

- Horse Power

- Speed Range

- Feed Range

- Work Envelope

- Accuracy.

- Tool change time

- Feed change time

- Speed change time

- Table rotation time

- Tool adjusting time

- Rapid traverse time. 
After the required data have been entered, the CAPP users give the machine a released status. Once again, the necessary checks as to the completeness of the data are carried out. If all required information has been input, the release is successful.

This scenario shows the activity of creating a new workcenter in the system. Other scenarios involving workcenters, such as deletions and reviews, are established in the same way. Whereas both CAPP and MRP-II can originate reviews, only MRP-II has the authority to delete workcenters.

\section{2 .3 REVISIONS OF PURCHASED TOOL PARTS FROM CAPP}

Figure 5 shows the case where a revision of a purchased part is initiated within CAPP. When the revision of a new purchased part is first entered into the system from CAPP, it is assigned the following.

- Part Number

- Revision level

- Status code

Initially this revision is in the working status in CAPP (state a) in the status diagram. This is the stage where details and specifications of the new revision are worked upon. CAPP then releases the new revision. A check is made to see if the part number exists. If it does, the release is successful.

The new skeletal revision record is created in $C A D$, with working status. CAD now works on the revision, and fills in the remaining information in its part revision record.

CAD gives the new revision a released status. This results in the automatic generation of the skeletal revision record in MRP-II, in hold status. If this is successful, then the release 
in CAD is successful.

Wher MRP-II fills in all the required information, it gives the revision a released status (state $g$ ). A check is made to see if the revision has a released status in CAPP. If it has, then the release is successful in MRP-II. Before entering a new revision into the system, CAPP must place the old revision on hold. This is shown in figure 6. This action triggers a hold in both CAD and MRP-II respectively.

Revisions of parts can be originated from any of the three functions. In case of manufactured parts, new routings would be required, whereas routings are not required for purchased parts. Non-product parts such as those which originate from MRP-II, can have revisions too. Once again the other scenarios relating to revisions can be constructed, with changes in the sequence of events.

\section{DISCUSSION}

The facility level CIM system described above is generic. It is not claimed that it can act as a universal bridge box, whereby any existing CAD, CAPP, or MRP-II system can be attached, and be fully integrated. In actual implementation, some features of this system will have to be incorporated within the stand alone application systems themseleves. For example, the incorporation of the facility of having status codes, if not already available, in the specific application software. Some existing software packages do support status codes, whereas others will have to be modified to do so for successful 
implementation. So in effect, the model stands as a controller or driver of the proposed C.I.M. system. Because of the variety of software available in each of these three areas, the software within a given area differ greatly in terms of format and architecture. So integrating a specific set of software, will involve minor modifications to the model, so as to take into account these differences. That is why the effort has been to keep this model as generic as possible. A weakness has been the current modeling and analysis technique. It is not a formal approach, and lacks the ability to show the sequence and parallelism of events in the scenarios. More detailed and formal modeling techniques such as Petri nets (Peterson 1977) are currently being investigated.

\section{MULTI DATABASE INTEROPERABILIX.}

To implement and demonstrate the integrated MRP II/CAD/CAPP system, the concept of database interoperability is being utilized. It is based on the Update Dependency language, currently under development in the department of computer Science at the University of Maryland, as a tool for achieving interoperability (Mark 1987). The language is being applied to the current problem as a means to critically analyze the design of the integrated system, as well as to analyze the effectiveness of the language in specifying such a system. Therefore, a rule set is constructed for the integrated system, called update and retrieval dependencies, which controls inter-database consistency through inter-database operation calls. This rule set will be used here to implement the integrated MRP-II/CAD/CAPP system. 


\section{CONCLUSIONS}

It is visualised that this generic integration model could be used as a framework, within which individual integration implementations are accomplished. Current integration efforts are largely spent towards direct $C A D$ and $C A M$ integration. This is not what is viewed as a true integrated system. According to a popular definition of CIM, ie; " CIM is the harnessing of all information required to correctly produce products that comply with the business plan of the enterprise" (Schnur 1987), CAD and CAM integration is but a small part of the entire integration effort. The approach in this work is to show that CIM is possible. Moreover, it does not require complete redesign, with accompanied hardware and software replacements. It can be modular and in most cases transprent to the user.

ACKNOWLEDGEMENTS The System Research Center of the University of Maryland, is acknowledged for funding the database interoperability part of this research. (GRANT NO: NSFD CDR-85-00108)

\section{REFERENCES}

Fox, Kenneth A., 1984, MRP II Providing a Natural 'Hub' for Computer Integrated Manufacturing systems. Industrial Engineering vol. 16, no.10, 44-50.

Harhalakis, G., et al. 1987, An Integration of Manufacturing Resource Planning (MRP II) Systems and Computer Aided Design (CAD) Based on Update Dependencies. International conference on Data and Knowledge systems for Manufacturing and 
Engineering, Hartford, Connecticut.

Harhalakis, G., et al. 1986. Integration of Manufacturing Resource Planning (MRP II) systems with Computer Aided Design (CAD). Proceedings of the 13th Conference on Production Research and Technology, organised by the National Science Foundation, Gainsville, Florida. Research and Technology Program, 1986.

Mark, I., et al. 1987. Operational specification of Update Dependencies. Department of Computer science, University of Maryland.

Melkanoff, M.,1984, The CIMS Database: Goals, Problems, Case Studies, and Proposed Approaches outlined. Industrial Engineering, vol. 16, no.11, 78-92.

Peterson, James L.,1977. Petri Nets. Computing Surveys, vol. 9. No.3.

Schnur, Joel A., 1987. Can there be CIM without MRP II? CIM Review vol.3, no. 4, 3-6.

Ssemakula, M.E., 1987. The role of process planning in the integration of CAD/CAM systems. Proceedings, Fourth European Conference on Automated Manufacturing (AUTOMAN 4), Birmingham, U.K. 


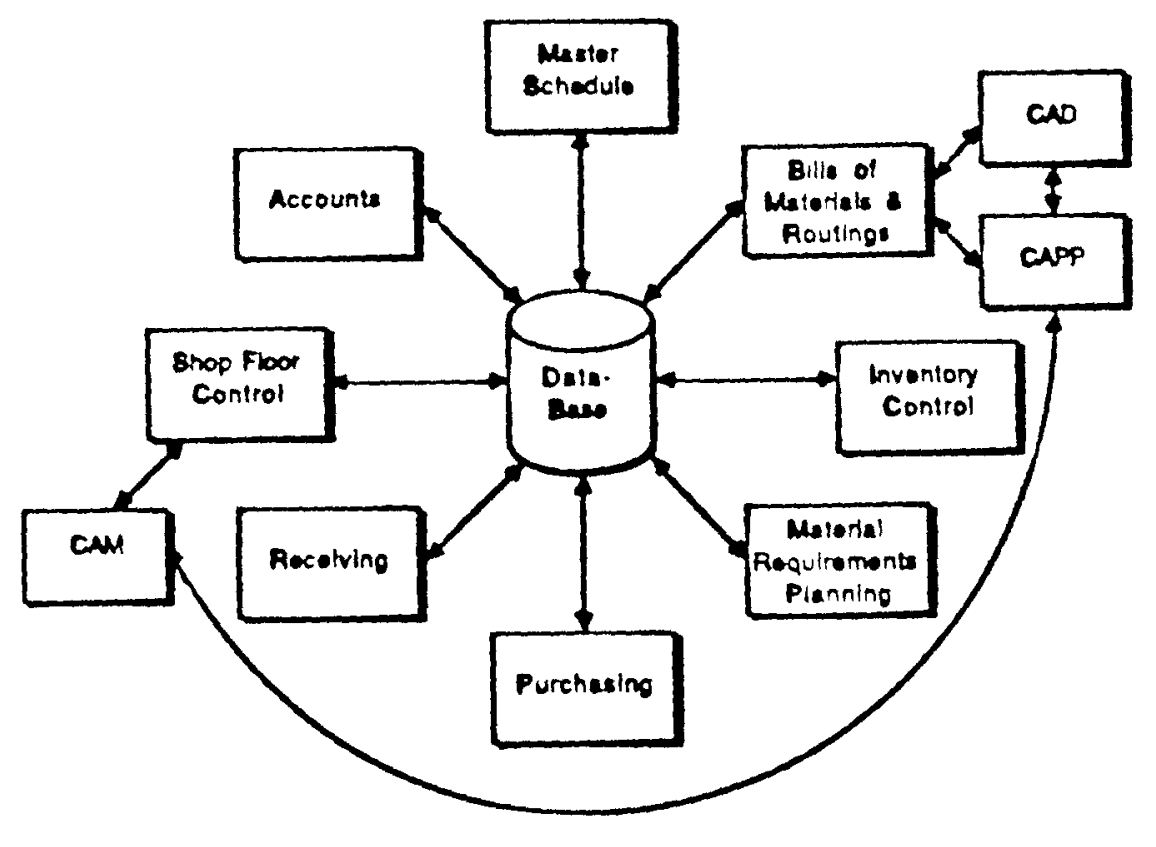

FIG 1 


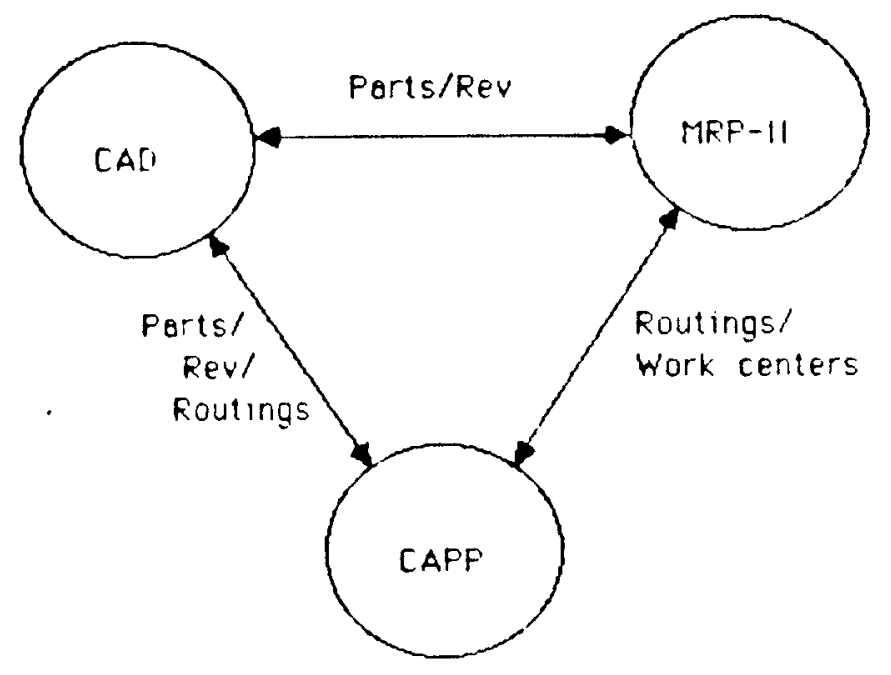

FIG 2 . 

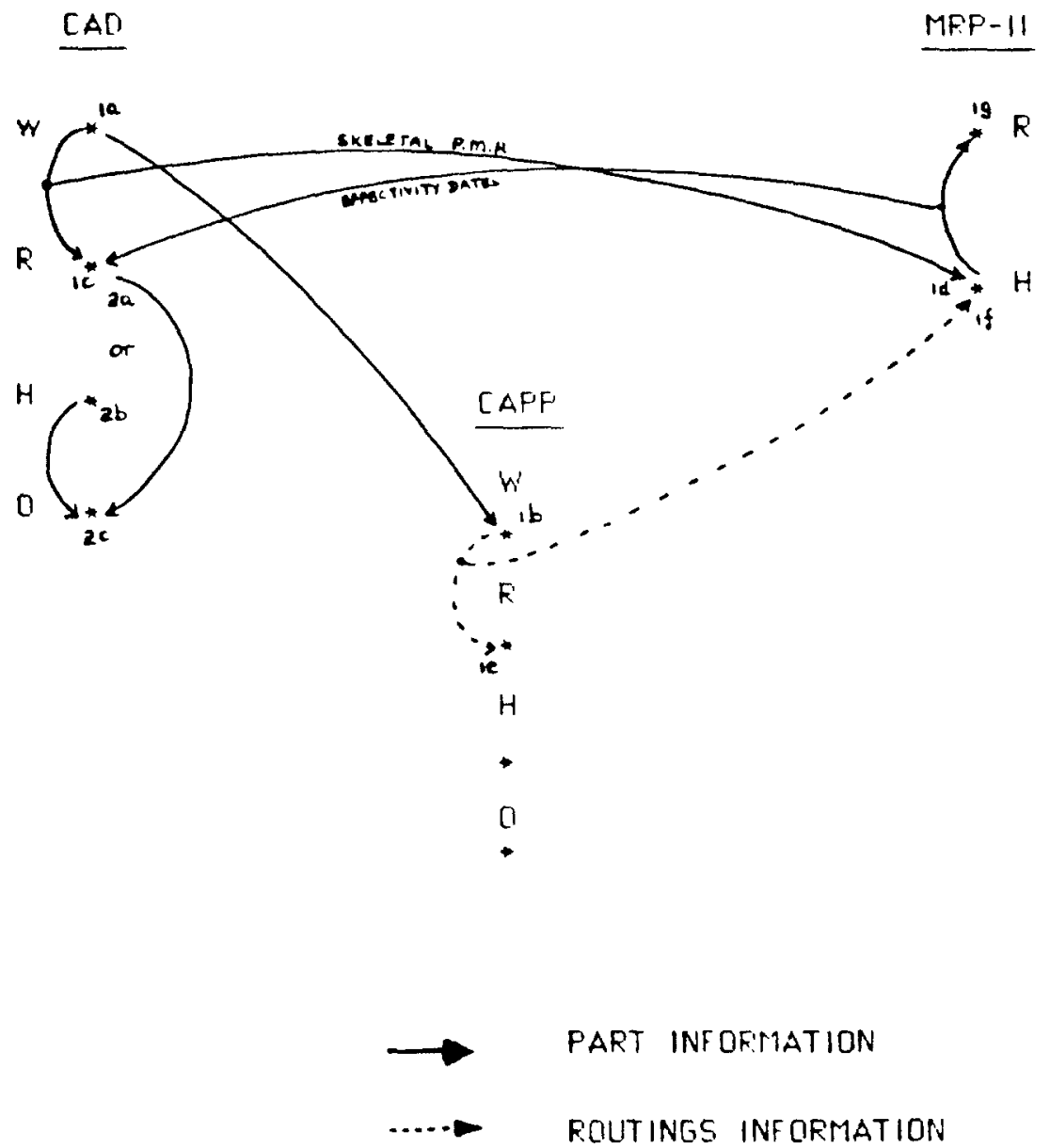

FIG 3. 


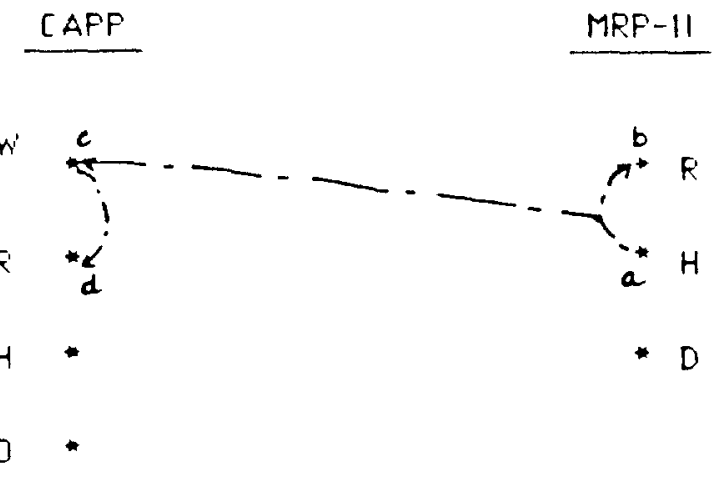

$\rightarrow$ WOFICENTEF IISFGFMATION

FIG. 4 


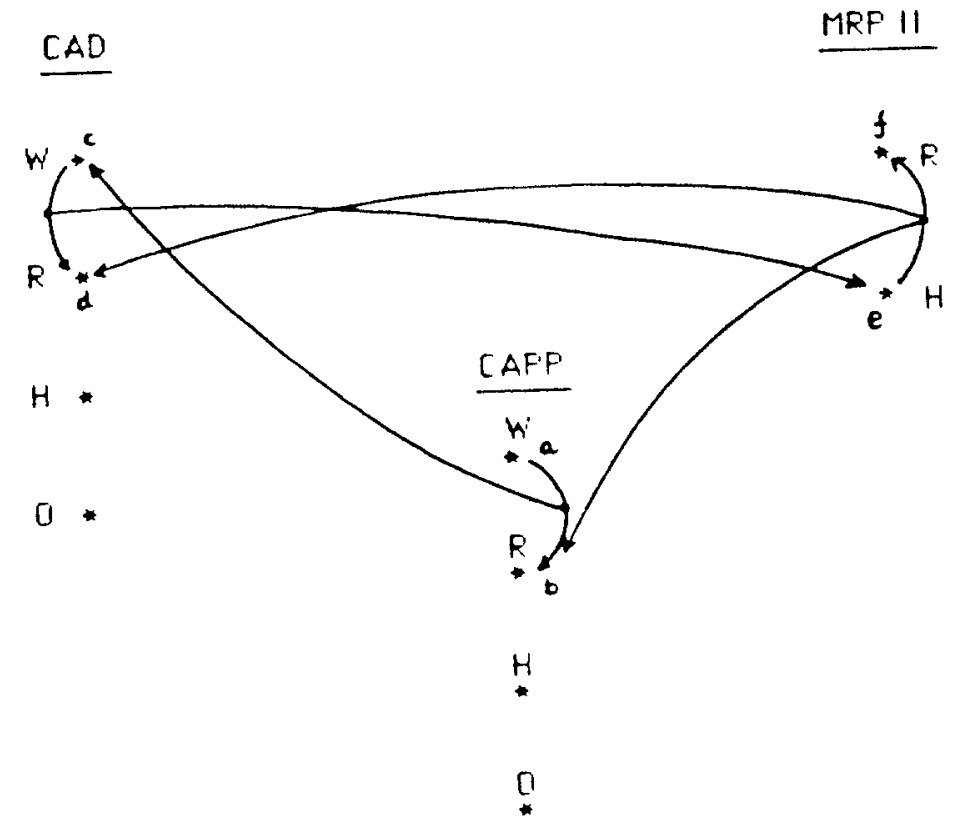

$\rightarrow$ faft inflamiation

FIG 5 


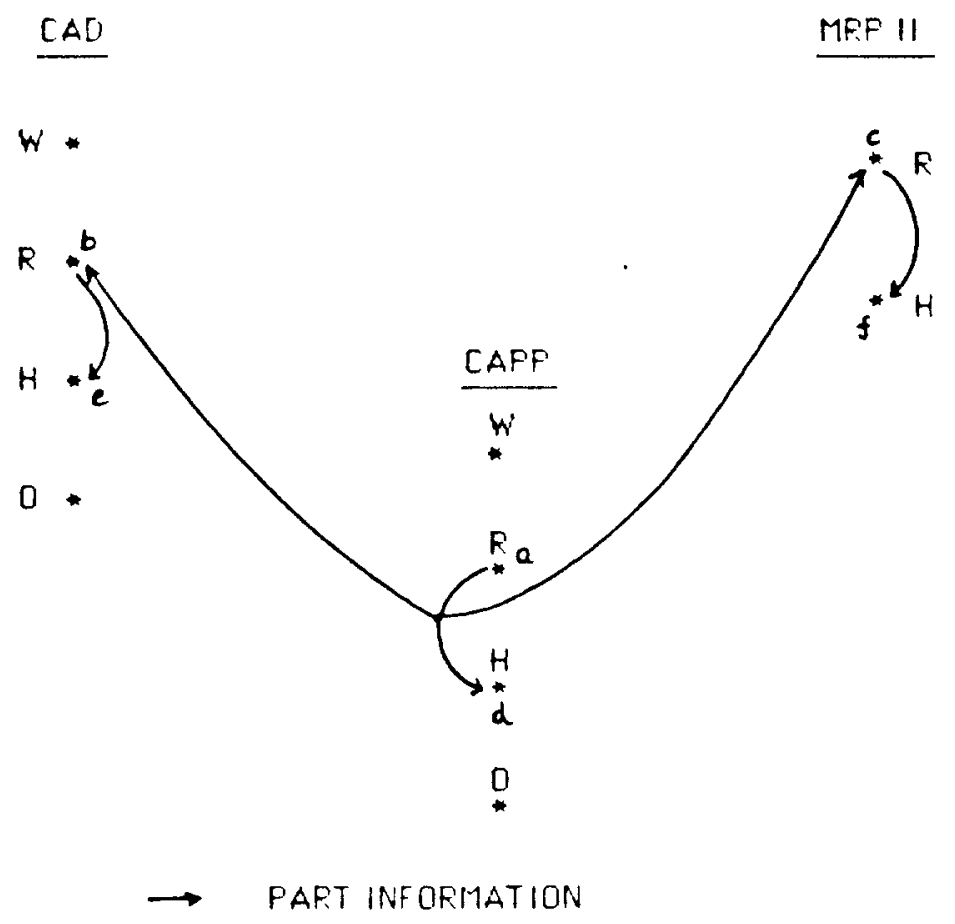

FIG 6 


\section{ILLUSTRATIONS}

figure 1: MRP-II as the hub of a C.I.M. system.

figure 2: Information flow between the three functions.

figure 3: New manufactured product part from CAD.

figure 4: Installation of a New workcenter.

figure 5: Revisions of purchased parts from CAPP (new revision).

figure 6: Revisions of purchased parts from CAPP (old revision). 